(C) [2009] IEEE. Reprinted, with permission, from Lei, Gang., Shao, Ke., Guo, Youguang., Zhu, Jianguo \& Lavers, J 2009, mproved Sequential Optimization Method for High Dimensional Electromagnetic Optimization Problem', IEEE Transactions On Magnetics, Vol. 45, no. 10, pp. 3993-3996. This material is posted here with permission of the IEEE. Such permission of the IEEE does not in any way imply IEEE endorsement of any of the University of Technology, Sydney's products or services. Internal or personal use of this material is permitted. However, permission to reprint/republish this material for advertising or promotional purposes or for creating new collective works for resale or redistribution must be obtained from the IEEE by writing to pubs-permissions@ieee.org. By choosing to view this document, you agree to all provisions of the copyright laws protecting it. 


\title{
Improved Sequential Optimization Method for High Dimensional Electromagnetic Device Optimization
}

\author{
Gang $\mathrm{Lei}^{1}$, K. R. Shao ${ }^{1}$, Youguang Guo ${ }^{2}$, Jianguo $\mathrm{Zhu}^{2}$, Senior Member, IEEE, and J. D. Lavers ${ }^{3}$, Fellow, IEEE \\ ${ }^{1}$ College of Electrical and Electronic Engineering, Huazhong University of Science and Technology, Wuhan, 430074, China \\ ${ }^{2}$ Faculty of Engineering, University of Technology, Sydney, N.S.W. 2007, Australia \\ ${ }^{3}$ Department of Electrical and Computer Engineering, University of Toronto, Toronto, ON M5S 3G4, Canada
}

\begin{abstract}
Improved sequential optimization method (SOM) and dimension reduction optimization method (DROM) are presented for high dimensional optimization design problems of electromagnetic devices. Improved SOM can simultaneously optimize the statistical approximate models and optimization algorithms more efficiently compared with SOM. Using DROM, a high dimensional problem can be converted into a low dimensional problem with expert experience or some design of experiment techniques. Then two TEAM benchmark problems (Problem 22 and Problem 25) are investigated to illustrate the efficiency of the proposed methods. From the experimental results, we can see that the presented methods can obviously reduce the computational cost of finite element analysis, while the optimal results also satisfy design specification.
\end{abstract}

Index Terms - Analysis of variance, DROM, electromagnetic devices, improved SOM.

\section{INTRODUCTION}

$\mathrm{M}$ any electromagnetic devices are designed by means of finite element models with direct optimization algorithms. However, the higher the accuracy of design objective is, the more expensive direct optimization is expected to be. Sometimes, this cost may be prohibitive. As an alternative, many statistical approximate models are employed to ease computational burden of direct optimization method. They are proved fast, but not very accurate. Furthermore, as models and algorithms were almost discussed separately in traditional optimization methods, and this may induce the waste of computation cost [1], [2].

In order to make up for the low fidelity of approximate model and the expensive cost of optimization algorithm, we have introduced sequential optimization method (SOM) to low dimensional design problems [2]. SOM can optimize the approximate model and algorithm simultaneously. However, SOM is difficult to deal with high dimensional design problems. For example, the points of five level full factorial design in the model construction might be impractical if there were seven variables to study instead of just three. Therefore, it is essential to develop a set of new methods for high dimensional design problems.

\section{IMPROVED SOM}

Fig. 1 is the flowchart of improved SOM which includes six main steps. Improved SOM is also composed of coarse optimization process (COP: step 2, 3 and 4) and fine optimization process (FOP: step 5 and 6). The main purpose of the former is to reduce the design space, while the target of the latter is to update the optimal design parameters. Table I lists all the relevant abbreviations used in this paper.

Digital Object Identifier inserted by IEEE

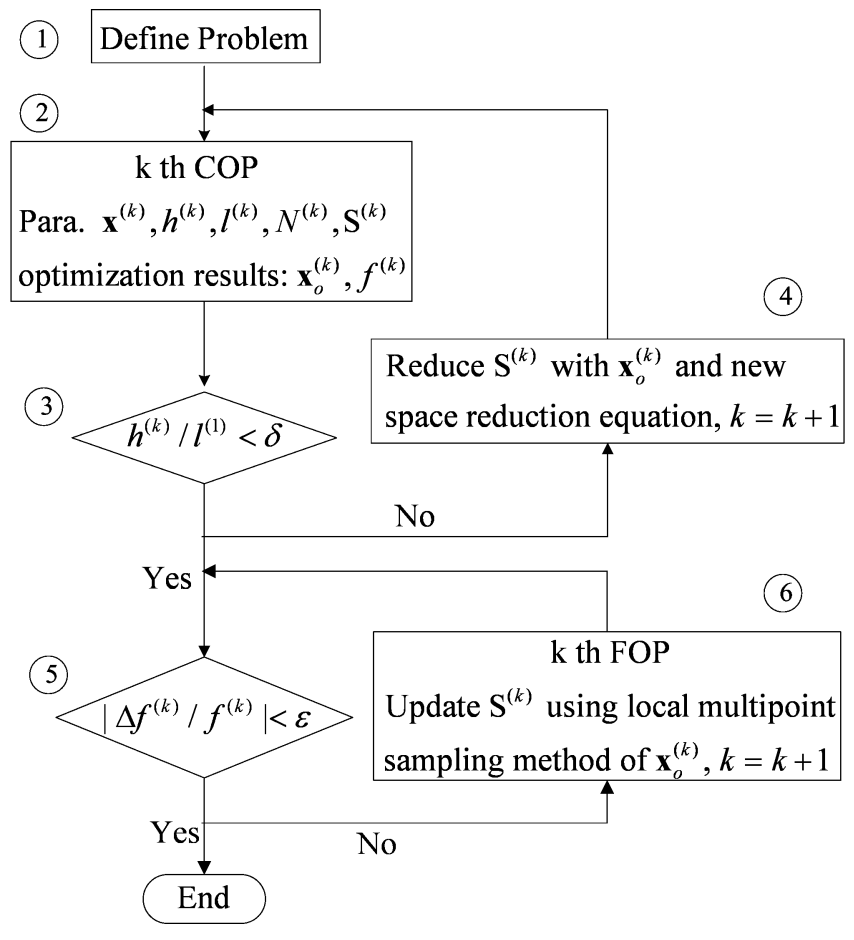

Fig. 1. The flowchart of improved SOM

TABLE I

ABBREVIATIONS AND DEFINITION

\begin{tabular}{|c|l|}
\hline Abbreviation & Definition \\
\hline ANOVA & Analysis of variance \\
COP & Coarse optimization process \\
DEA & Differential evolution algorithm \\
DROM & Dimension reduction optimization method \\
FESP & Finite element sample points \\
FOP & Fine optimization process \\
SMES & Superconducting magnetic energy storage \\
SOM & Sequential optimization method \\
\hline
\end{tabular}


Kriging model is selected as the approximate model in this work. Essentially, it is a multivariate statistical regression model. It has been widely applied to the design problems of electromagnetic devices [3], [4].

In the Fig. $1, \mathbf{x}^{(k)}$ is design space of the $k$ th optimization process, $l^{(k)}$ is interval, $h^{(k)}$ is step size, $N^{(k)}$ is the number of sample points and $\mathrm{S}^{(k)}$ is sample set. $\mathbf{x}_{o}^{(k)}$ and $f^{(k)}$ are the optimization results of Kriging model which is constructed with $S^{(k)}$. Differential evolution algorithm (DEA) is selected as the optimization algorithm in this work. The main DEA parameters are set as: mutation scaling factor is 0.8 , crossover factor is 0.8 , population number is $5^{*} D$ ( $D$ is the dimension of the designed problem), and the maximum stall generation is 50, which is selected for the stop criterion [5]. Four main improvements have been made in the improved SOM.

The first improvement is new space reduction equation in the COP. Generally, we reduce the sampling space after each COP to get the most interesting design space. Under the boundary condition of the design space, the design space of next step is updated as follows.

$$
\begin{aligned}
& x_{l i}^{(k+1)}=\max \left\{x_{l i}^{(k)}, \text { round }\left[\left(x_{o i}^{(k)}-\Delta l\right) / \Delta h\right] \Delta h\right\}, \\
& x_{u i}^{(k+1)}=\min \left\{x_{u i}^{(k)}, \text { round }\left[\left(x_{o i}^{(k)}+\Delta l\right) / \Delta h\right] \Delta h\right\},
\end{aligned}
$$

where $\left[x_{l i}^{(k)}, x_{u i}^{(k)}\right]$ is boundary of the $i$ th variable, function $\operatorname{round}(x)$ rounds $x$ to the nearest integer. $\Delta l=l_{i}^{(k)} / n l$ and $\Delta h=h_{i}^{(k)} / n h$, where $n l$ and $n h$ are reduction factors.

For a practical problem, there are three parameters $(n l, n h$ and $N$ ) to determine for the construction of Kriging model. $\mathrm{nl}$ can be 4,6 or 8 , the corresponding intervals of reduced space are $1 / 2,1 / 3$ and $1 / 4$ that of the former space. $n h$ can be 2 , 4 , or 8 , which let function $\operatorname{round}(x)$ to round $x$ with $1 / 2,1 / 4$ and $1 / 8$ of the current step size. $N$ may be 2, 3, 4 or 5 for a standardization space of $[0,1]$. Now, we use Monte Carlo method to give a selection strategy for these parameters.

Supposing design space is $[0,1]$ and $n l$ is 4 , we use Monte Carlo method to generate $10^{6}$ random numbers as the optimal results of Kriging model. Then we use these numbers to reduce the design space under $N=\{2,3,4,5\}$ and $n h=\{2,4,8\}$. The target of this analysis is to compare the mean of the error between the current optimal results and the mean of the reduced space. Table II shows the results.

From Table II, we can see that the mean error decreases with the increase of $N$ and $n h$. The same results can be derived with $n l=6$ and $n l=8$. So in our latter implementation, the default value of $n h$ is 8 and $N$ is 5 . For the factor $n l$, it must be selected with respect to the practical problems. If we have some experience about the problem or the given space is very large and we want to reduce the design space more quickly, $n l=6$ or 8 can be selected in the former COP. And if we have no prior experience, $n l=4$ may be a better choice.
From the table, we can see that this strategy is very important for the case of $N=2$. For example, supposing the initialization space is $[0,1], n h$ is 2 , and the current optimal

TABLE II

MEAN ERROR OF SPACE REDUCTION EQUATION

\begin{tabular}{|c|c|c|c|}
\hline$N$ & $2(n h)$ & $4(n h)$ & $8(n h)$ \\
\hline 2 & 0.1249 & 0.0624 & 0.0469 \\
\hline 3 & 0.0625 & 0.0469 & 0.0390 \\
\hline 4 & 0.0555 & 0.0416 & 0.0364 \\
\hline 5 & 0.0469 & 0.0391 & 0.0352 \\
\hline
\end{tabular}

value is 0.5 , from (1) and (2) we can get the next sample space is $[0.5,1.0]$. And if $n h$ is 8 , the next sample space is $[0.25$, $0.75]$. So we can minimize the distance between the optimal results and the mean of the next design space with this improvement. And this is very important for dimension reduction process of high dimensional problems.

The second improvement is the disposal of the boundary conditions. There are many discrete design spaces in practical problems, such as the poles of motor. When this case happens, the next design space in (1) and (2) should be rounded to the nearest feasible design points.

The third improvement is the local multipoint samples updating method in the FOP. To avoid the matrix to be singular in the modeling process, a least interval about the updated sample points is set. When the relative norm of two points is less than 0.01 , the latter point is deleted.

The forth improvement is the selection of optimal results. As Kriging model is only an approximation of the response surface, so we must compare the results given by optimization algorithm and those sampled in modeling, and the better one is selected as the optimal results for this optimization process.

Furthermore, for some objectives which can be expressed with analytical equations, such as mass, they should not be included in the approximate model to reduce the design error.

\section{DROM WITH IMPROVED SOM}

One difficulty with the former SOM is that the number of sample points in modeling increases exponentially with the number of variables you want to manipulate. For a 5-level full factorial design with 3 variables, $5^{3}=125$ finite element runs may be acceptable. For the 4 variables case, a full factorial design needs 625 finite element runs, this may be too much for many problems. And for a higher dimensional problem (more than 4 variables), it is unpractical to design a 5-level full factorial experiment. Therefore, it is necessary to develop new method for high dimensional design problems.

The optimization process of high dimensional problems consists of dimension reduction optimization process and sequential optimization process. The implementation of the former process is briefly described as follows.

1) Sample some points from direct optimization algorithm (about 40 iterations). It should be noted that a least interval about the two sample points is also set in the sampling process 
to avoid the matrix to be singular in the in the reconstruction of approximate model.

2) Construct the approximate model with the points sampled above, optimize it and get optimal results. Meanwhile, the sampled points should also be considered. So the optimal results in this step are the better one of that given by optimization algorithm and those sampled in modeling.

3) Reduce the design space with the current optimal results and new space reduction equation.

4) Select the significant factors with two methods, one is expert experience, the other is the analysis of variance (ANOVA) technique. Expert experience includes experience of designers or experts, model mechanism analysis and so on. ANOVA is one of the most widely used statistical techniques to determine significant factors and their significant order in a multivariate problem [6]. ANOVA can be easily implemented with MATLAB software which is also used in this work.

With the above 4 processes, a high dimensional design problem can be reduced as a low dimensional design problem, and then improved SOM can be addressed to solve this problem. We call this method as the dimension reduction optimization method (DROM).

\section{EXPERIMENTS}

\section{A. IEEE TEAM Workshop Problem 25}

It is a benchmark problem for the optimization of die press model [7], [8]. Fig. 2 is central geometry configuration of die press model which includes four design parameters $\left(R_{1}, L_{2}, L_{3}\right.$, $L_{4}$ ) to maintain the radial magnetic induction equal to $0.35 \mathrm{~T}$. The objective function is the square error of the calculated and specified values (10 equidistant points on line $\mathrm{e} \sim \mathrm{f}$ ). As the objective function value is very small (about the order of $10^{-4}$ ), and this may reduce the sensitivity and distinguishability of the model reconstruction, so the objective function is given by

$$
W(\vec{x})=\left(\sum_{i=1}^{10}\left[\left(\mathrm{~B}_{\text {xip }}-\mathrm{B}_{\text {xio }}\right)^{2}+\left(\mathrm{B}_{\text {yip }}-\mathrm{B}_{\text {yio }}\right)^{2}\right]\right)^{1 / 4} \text {, }
$$

where subscripts $\mathrm{p}$ and o mean the calculated and specified values respectively.

\section{B. IEEE TEAM Workshop Problem 22}

This is also a benchmark problem for the optimization of superconducting magnetic energy storage (SMES) [9]-[11]. Fig. 3 shows the design model. All parameters should be optimized to minimize the mean stray fields $\left(B_{\text {stray }}\right)$ while keeping the stored energy close to $180 \mathrm{MJ}$. $B_{\text {stray }}$ is root mean square of 21 equidistant points on lines $a$ and $b$.

There are many ways to define the objective function for this problem. In this work, it is given by

$$
f(\vec{x})=B_{\text {stray }} / B_{\text {norm }},
$$

where $B_{\text {norm }}=3 \mathrm{mT}$. And three constraints are

$$
\begin{aligned}
& h(\vec{x})=|E / 180-1|=0, \\
& g(\vec{x})=\left|B_{\max }\right|-\min \left[\left(54-\left|J_{i}\right|\right) / 6.4\right] \leq 0, i=1,2, \\
& q(\vec{x})=R_{1}+d_{1} / 2+d_{2} / 2-R_{2}<0,
\end{aligned}
$$

where $E$ is the stored energy of SMES. The first constraint concerns the relative error of stored energy and the second is a quench condition that guarantees superconductivity. The third

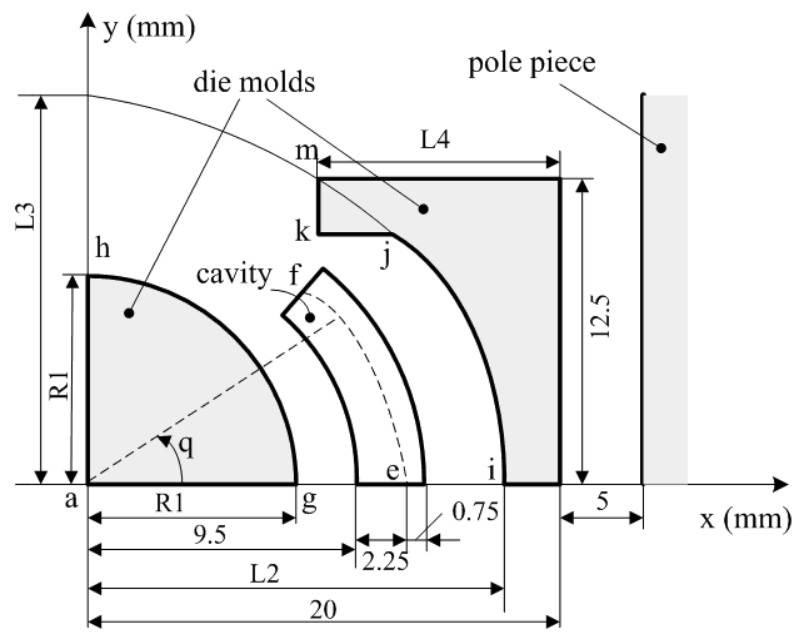

Fig. 2. The central configuration of die press model

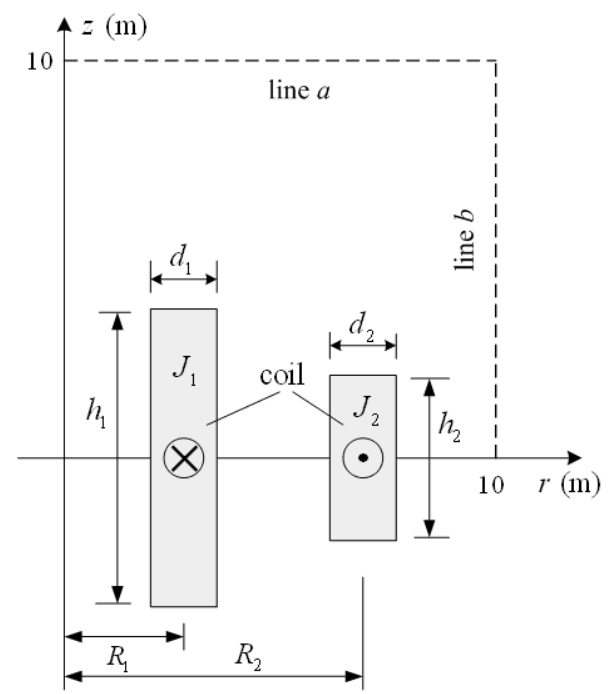

Fig. 3. The geometry configuration of SMES

is a geometric constraint which ensures that the two coils are not superposition. In order to reduce the unnecessary finite element analysis, this constraint is only used in the population initiation of the optimization process to select appropriate sample points. In this work, the former two constraints were treated using a penalty function as

$$
F(\vec{x})=f(\vec{x})+1000\left[h(\vec{x})^{2}+\max (g(\vec{x}), 0)^{2}\right] .
$$

\section{RESUltS}

The following results are given by our proposed methods. SOM parameters are $\delta=\varepsilon=5 \%$. To illustrate the efficiency of the proposed methods, the results given by direct optimization method are also given for each problem. In order to make all these results to be comparable, all response values, such as $B$ and $E$, are calculated from the ANSYS with the same preprocessing process (e.g. mesh method). 


\section{A. IEEE TEAM Workshop Problem 25}

In the implementation of this problem, 800 points were selected from DEA to construct the Kriging model. And from some former discussion, we can select $\left(R_{1}, L_{2}, L_{4}\right)$ as the three significant factors about this problem [7], [8]. That is to say, expert experience is used here for the determination of significant factors. In fact, we can get the same result with ANOVA technique [6], [8]. From the analysis, two main conclusions can be drawn as follows.

1) For the direct optimization method, the optimal results given by DEA are [8.0831, 16.3503, 14.9946, 15.1451], and the square error is $2.8^{*} 10^{-4}$. To get these results, 4320 finite element sample points (FESP) are needed.

2) For the optimization with the new methods, only two COPs are implemented to get the optimal results, which are [7.70, 15.525, 15.31, 15.0]. And to be more interesting, only 970 FESP are needed to get these results. The number of FESP is about $22.45 \%$ (less than 1/4) compared with that of DEA. And the square error is $8.3^{*} 10^{-4}$, which is a little bigger than that of DEA, but also satisfies the design specifications.

\section{B. IEEE TEAM Workshop Problem 22}

In the implementation of this problem, 1200 points was selected from DEA to construct the Kriging model. And the above two strategies may also be used to select the significant factors. In this work, we use ANOVA technique to determine the significant factors with MATLAB software. ANOVA indicates that the dimension of the outer coil $\left(R_{2}, h_{2}, d_{2}\right)$ can be selected as the significant factors. And their significant order is $R_{2}, d_{2}$ and $h_{2}$. Then we can convert an 8 parameters problem to a 3 parameters case with the significant factors. In fact, the selection results are also reasonable from the discrete case description of three-variable SMES [11].

Table III shows the optimal results given by our proposed methods. Two main conclusions can be drawn from the table.

1) For the direct optimization method, 4720 FESP are needed to get the optimal results. $B_{\text {stray }}$ is $2.2725 \mathrm{mT}$ and the error of energy is $1.25 \mathrm{MJ}$. $B_{\max }$ is $4.12 \mathrm{~T}$, which is less than the maximum permissible value $(4.91 \mathrm{~T})$.

2) For the new methods, two COPs and one FOP are needed for the implementation of SOM. And only 1516 FESP, about $32.12 \%$ (less than 1/3) compared with that of DEA, are needed to get the optimal results, which are presented in the 'DROM' column in the table. $B_{\text {stray }}$ is $2.9226 \mathrm{mT}$, which is a little bigger than that of DEA. But the error of energy is $0.27 \mathrm{MJ}$ (or $0.15 \%$ ), which is better than that given by DEA.

\section{CONCLUSIONS}

Two DROM strategies and improved SOM are addressed to solve high dimensional electromagnetic design problems. Compared with the former SOM, four main improvements are given for the improved SOM.
TABLE III

OPTIMIZATION RESULTS OF SMES

\begin{tabular}{|c|c|c|c|}
\hline Var. & Unit & DEA & DROM \\
\hline$R_{1}$ & $\mathrm{~m}$ & 2.3816 & 1.2407 \\
\hline$R_{2}$ & $\mathrm{~m}$ & 3.3772 & 3.2520 \\
\hline$h_{1} / 2$ & $\mathrm{~m}$ & 1.1182 & 1.1312 \\
\hline$h_{2} / 2$ & $\mathrm{~m}$ & 0.3659 & 0.2134 \\
\hline$d_{1}$ & $\mathrm{~m}$ & 0.1884 & 0.6416 \\
\hline$d_{2}$ & $\mathrm{~m}$ & 0.6531 & 0.5250 \\
\hline$J_{1}$ & $\mathrm{MA} / \mathrm{m}^{2}$ & 22.5717 & 10.6325 \\
\hline$J_{2}$ & $\mathrm{MA} / \mathrm{m}^{2}$ & -11.0582 & -13.9904 \\
\hline$B_{\text {stray }}$ & $\mathrm{mT}$ & 2.2725 & 2.9226 \\
\hline$E$ & $\mathrm{MJ}$ & 178.75 & 179.73 \\
\hline$B_{\max }$ & $\mathrm{T}$ & $4.12(4.91)$ & $5.81(6.25)$ \\
\hline FESP & - & 4720 & 1516 \\
\hline
\end{tabular}

Obviously, the proposed methods are very successful in optimizing the die press model and SMES device. The finite element computational effort of the present methods is less than $1 / 3$ compared with that of direct optimization method, while the results also satisfy design specification. Furthermore, many other design techniques of experiment, approximate models and algorithms can be included in these methods.

ACKNOWLEDGMENT

This work was supported by the National Natural Science Foundation of China (NSFC) under Grant 50877029.

\section{REFERENCES}

[1] L. D. Wang and D. A Lowther, "Selection of approximation models for electromagnetic device optimization," IEEE Trans. Magn.,vol. 42, no. 2, pp. 1227-1230, 2006.

[2] Gang Lei, K. R, Shao, Youguang Guo, Jianguo Zhu, and J. D. Lavers "Sequential optimization method for the design of electromagnetic device,” IEEE Trans. Magn., vol. 44, no. 11, pp. 3217-3220, 2008.

[3] L. Lebensztajn, C. A. R. Marretto, M. C. Costa, and J. L. Coulomb, "Kriging: a useful tool for electromagnetic device optimization," IEEE Trans. Magn., vol. 40, no. 2, pp. 1196-1199, 2004.

[4] S. N. Lophaven, H. B. Nielsen, and J. Sondergaard, "DACE: A MATLAB Kriging toolbox version 2.0," Technical Report IMM-TR-2002-12, Technical University of Denmark, Copenhagen, 2002.

[5] R. Storn and K. Price, "Differential evolution-A simple and efficient heuristic for global optimization over continuous spaces," Journal of Global Optimization, vol. 11, pp. 341-359, 1997.

[6] C. F. J. Wu and M. S. Hamada, Experiments: Planning, Analysis and Parameter Design Optimization. Wiley, New York, 2000.

[7] L. Lebensztajn and J. L Coulomb, "TEAM workshop problem 25: a multiobjective analysis," IEEE Trans. Magn., vol. 40, no. 2, pp. 14021405, 2004.

[8] M. C. Costa, J. L. Coloumb, and Y. Maréchal, "Parameters screening of TEAM workshop problem 25 by the application of experimental design method," in Proc. ISTET 2001, Linz, Austria, Aug. 19-22.

[9] F. Campelo, F. G. Guimaraes, H. Igarashi, and J. A. Ramirez, "A clonal selection algorithm for optimization in electromagnetics," IEEE Trans. Magn.,vol. 41, no. 5, pp. 1736-1739, 2005.

[10] Gang Lei, K. R. Shao, Y. B. Li, G. Y. Yang, Jun Zhao, "Sequential response surface model method for the optimization design of SMES," in Proc. 11th ICEMS, China, Oct. 17-20, 2008, pp. 328-331.

[11] [Online]. Available: http://www.igte.tugraz.at/archive/team/index.htm. 